\title{
Dust heating in the cores of 3 CRR radio galaxies
}

\author{
M. Birkinshaw, D. M. Worrall and A. Bliss \\ HH Wills Physics Laboratory, University of Bristol \\ Tyndall Avenue, Bristol BS8 1TL, UK
}

\begin{abstract}
We have undertaken a Spitzer campaign to measure the IR structures and spectra of low-redshift 3CRR radio galaxies. The results show that the $3.6-160 \mu \mathrm{m}$ infrared properties vary systematically with integrated source power, and so demonstrate that contemporary core activity is characteristic of the behaviour of sources over their lifetimes. IR synchrotron emission is seen from jets and hotspots in some cases. Thermal emission is found from a jet/gas interaction in NGC 7385. Most of the near-IR integrated colours of the low-redshift 3CRR radio galaxies are similar to those of passive galaxies, so that IR colours are poor indicators of radio activity.
\end{abstract}

Keywords. galaxies: active, radio continuum: galaxies, infrared: galaxies

\section{Introduction}

We have undertaken an IR imaging campaign for the low-redshift 3CRR (Laing, Riley \& Longair 1983) radio sources using the IRAC (3.6, 4.5, 5.8, and $8.0 \mu \mathrm{m}$ band) and MIPS (24, 70, and $160 \mu \mathrm{m}$ band) cameras on the Spitzer satellite (Fazio et al. 2004; Rieke et al. 2004). Our main aims were (1) to quantify the extent to which signatures of the radio activity of the cores appear in the core IR emission; (2) to investigate the heating of dust and gas in the core caused by the active nucleus; (3) to investigate whether the host galaxies display any extended dust features, such as those that might arise from recent mergers or from uplifts from the core; and (4) to image IR emission from radio structures such as jets and hot spots, and so to study the relativistic electron populations that they contain.

\section{Spitzer imaging}

The sensitivity and angular resolution that the Spitzer cameras provide is a strong function of wavelength, and the images of the 3CRR galaxies usually show structure in the host galaxy only in the IRAC bands, where the FWHM of the point spread function (PSF) varies from 1.6 to 1.9 arcsec. The 6 to 40 -arcsec PSFs of the MIPS images provide structural information only for the closest objects, such as M 87 (=3C 274), but since the IR emission is usually concentrated in the cores in the MIPS bands, the uncertainty in the core flux densities introduced by our inability to make precise fits to the structures of the galaxies is not usually a limiting factor in measuring useful core spectra.

The basic reduction of the Spitzer data used IRACproc (Schuster, Marengo \& Patten 2006) and MOPEX (Makovoz \& Marleau 2005), and detailed image analysis and photometry were then performed using codes developed in Bristol. Photometry for point-like components is accurate to $5 \%$ to $20 \%$, depending on band, and photometric errors represented in Figures 1-3 below include both systematic and statistical components. These errors become large in the IRAC bands at the high-redshift limit of the study, $z=0.1$, because of poor structural discrimination between the cores and the host galaxies. The 

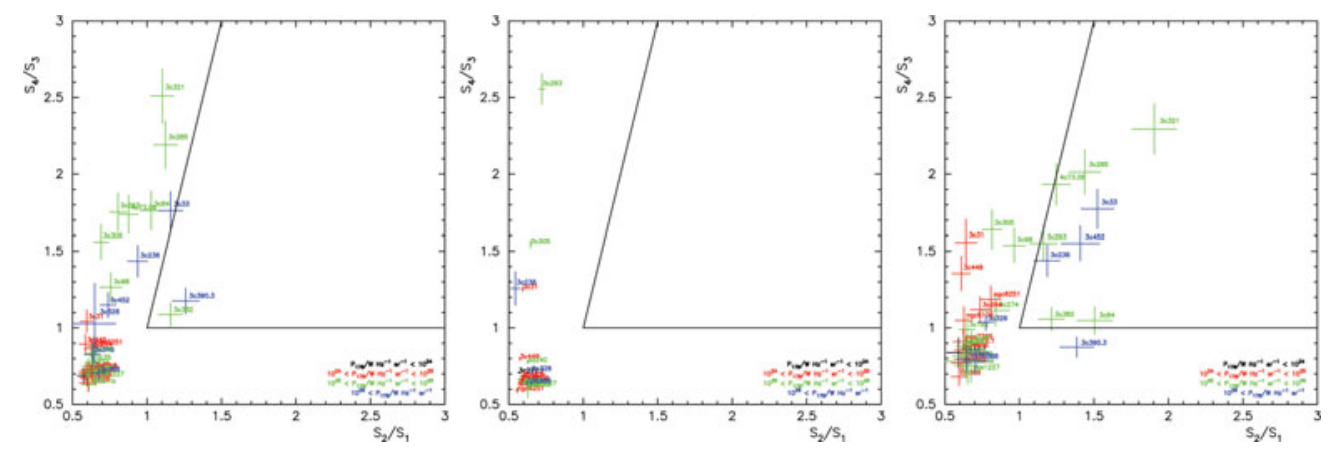

Figure 1. IRAC colours of the 3CRR radio galaxies, with the $S_{4} / S_{3} \equiv 8.0 / 5.8 \mu \mathrm{m}$ colour plotted against the $S_{2} / S_{1} \equiv 4.5 / 3.6 \mu \mathrm{m}$ colour. Left: measured in a fixed 21-arcsec radius aperture. Centre: total light from the host galaxy component, as determined from a composite Sérsic plus point-source model fit. Right: light from the core, excluding host galaxy light to the greatest extent possible. The lines on each panel separate the region occupied by active galaxies (upper right) from the region occupied by passive galaxies (lower left). These lines were derived from the selections of Stern et al. (2005) and Seymour et al. (2007). Only two 3CRR objects lie in the active-galaxy region in total light, and only about $30 \%$ could be identified as active based on their core colours. IR surveys are therefore poor at finding radio galaxies of low or intermediate power.

errors may also become large for faint emission in crowded fields, such as in fitting for the IR emission from the hot spots in 3C 33 (Kraft et al. 2007).

The IRAC structure of each host galaxy was fitted by an elliptical Sérsic model (Sérsic 1963) with a point-like component at its centre, convolved with a model PSF, and with foreground stars, jets, and overlapping galaxy images masked. This fitting procedure could also be used for the 24- $\mu \mathrm{m}$ MIPS band in some cases. For most of the MIPS images, however, a sufficiently good fit was obtained with a simple point source model because of the relatively large PSF. The signal/noise of the MIPS flux densities in the 70and $160-\mu \mathrm{m}$ bands is sometimes quite poor because of the lumpy background structure in the images.

Some residual images from the fitting showed clear signs of jets, for example in 3C 31 (Lanz et al. 2011), 3C 66B (earlier reported by Tansley et al. 2000 from ISO data), and NGC 6251. In the case of NGC 6251 the jet is seen to extend well beyond the optical image of the galaxy, and even beyond the IRAC frame. When the residual images were compared with the radio structures other features sometimes became apparent, such as IR-bright hotspots in cases such as 3C 33 (Kraft et al. 2007), and jet interactions, such as the prominent examples in NGC 7385 (Rawes et al. 2014) and 3C 390.3.

\section{Spectra}

The colours of the $353 \mathrm{CRR}$ radio galaxies in the sample are shown in Fig. 1. The lefthand panel shows the IRAC $S_{4} / S_{3} \equiv 8.0 / 5.8 \mu \mathrm{m}$ colours as a function of the $S_{2} / S_{1} \equiv$ $4.5 / 3.6 \mu \mathrm{m}$ colours, measured over a fixed 21 -arcsec radius aperture. For only two cases do these colours suggest the presence of an active galaxy, according to the criteria of Stern et al. (2005) or Seymour et al. (2007). That is, the average 3CRR radio galaxy at low redshift appears passive. The exceptions are the two broad-line objects in the sample, 3C 382 and 3C 390.3. This is as expected, since the Stern et al. and Seymour et al. selection cuts were designed to find broad-line objects. The high $8.0 / 5.8 \mu \mathrm{m}$ ratios evident for some of the galaxies could be interpreted as the signature of strong PAH 


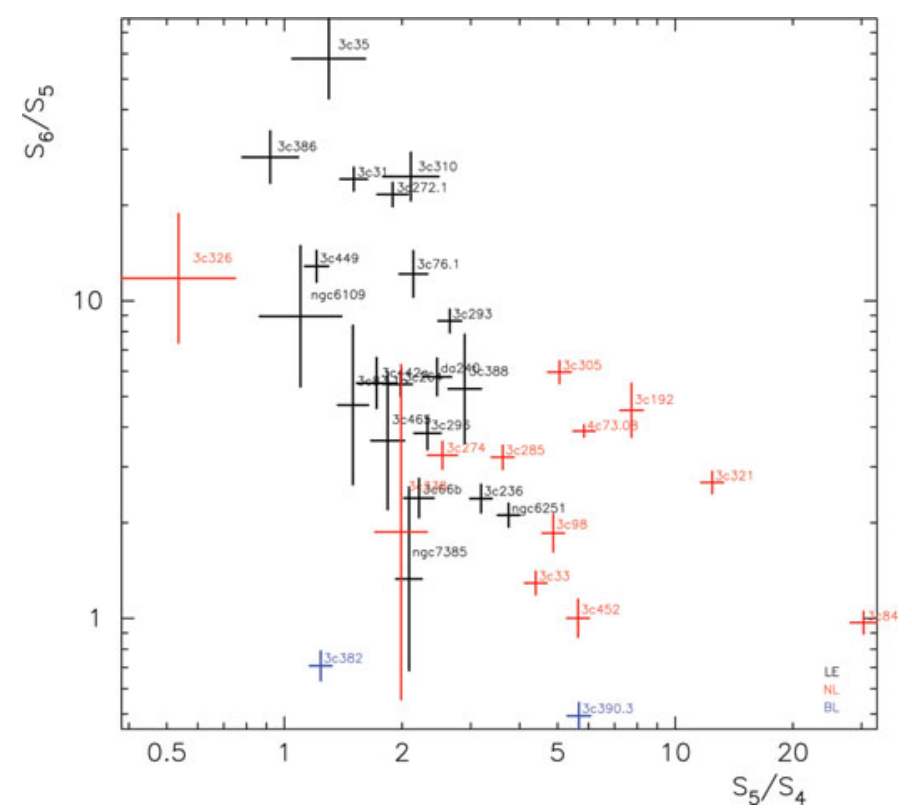

Figure 2. Combined MIPS and IRAC colours for the cores of the 3CRR radio galaxies. The ratio of $70 \mu \mathrm{m}$ and $24 \mu \mathrm{m}$ fluxes $\left(S_{6} / S_{5}\right)$ is shown as a function of the ratio of $24 \mu \mathrm{m}$ and $8 \mu \mathrm{m}$ fluxes $\left(S_{5} / S_{4}\right)$. The position of a radio galaxy on this diagram is well-correlated with its emission-line type, except that the narrow-line source 3C 326 appears anomalous, since it lies in the region occupied by low-excitation objects.

emission rather than an active nucleus. Many of the higher-power sources in the sample remain in the region characteristic of pure stellar continuum, which is close to $(0.7,0.8)$ in this colour-colour plot.

The colours of the host galaxies are shown in the centre panel of Fig. 1. This clearly shows the passive nature of the hosts - the concentration into the region of passive galaxy colours is more obvious. The most extreme departure from the locus of passive galaxies occurs for 3C 293, which is known to be gas-rich.

When the light from the host galaxies has been removed more of the 3CRR galaxy nuclei show themselves to be active. The core colours are shown in the right-hand panel of Fig. 1. The Spitzer PSF corresponds to a scale of more than $3 \mathrm{kpc}$ at the highest redshift in this sample, and so the central stellar emission in the host galaxy is still a major component of the emission in the IRAC bands. This affects the IRAC colours, and the activity of the nuclei is only apparent for those cores which exhibit a strong warm-dust emission component. Broad-line objects (such as 3C 382), narrow-line objects (such as 3C 285), and low-excitation objects (such as 3C 236) can now be found in the Stern et al. and Seymour et al. active-galaxy selection region. However, about $70 \%$ of the $3 \mathrm{CRR}$ radio galaxies would not be selected by near-IR colour, even based on their core colours. Improving the linear resolution for measurement of the IR colours of the cores would make little difference - the nearby 3CRR objects for which the Spitzer PSF corresponds to sub-kpc linear scales are not notably better separated from the locus of the passive galaxies than are the more distant objects.

The line-emission characteristics of the sources are better distinguished in colours based on a combination of the MIPS and IRAC bands, as in Fig. 2. The two broad-line emitting objects lie at the bottom of the diagram, and the low-excitation sources tend to lie in the upper left, with the narrow-line emitting cores in the lower right. 3C 326 is anomalous, 


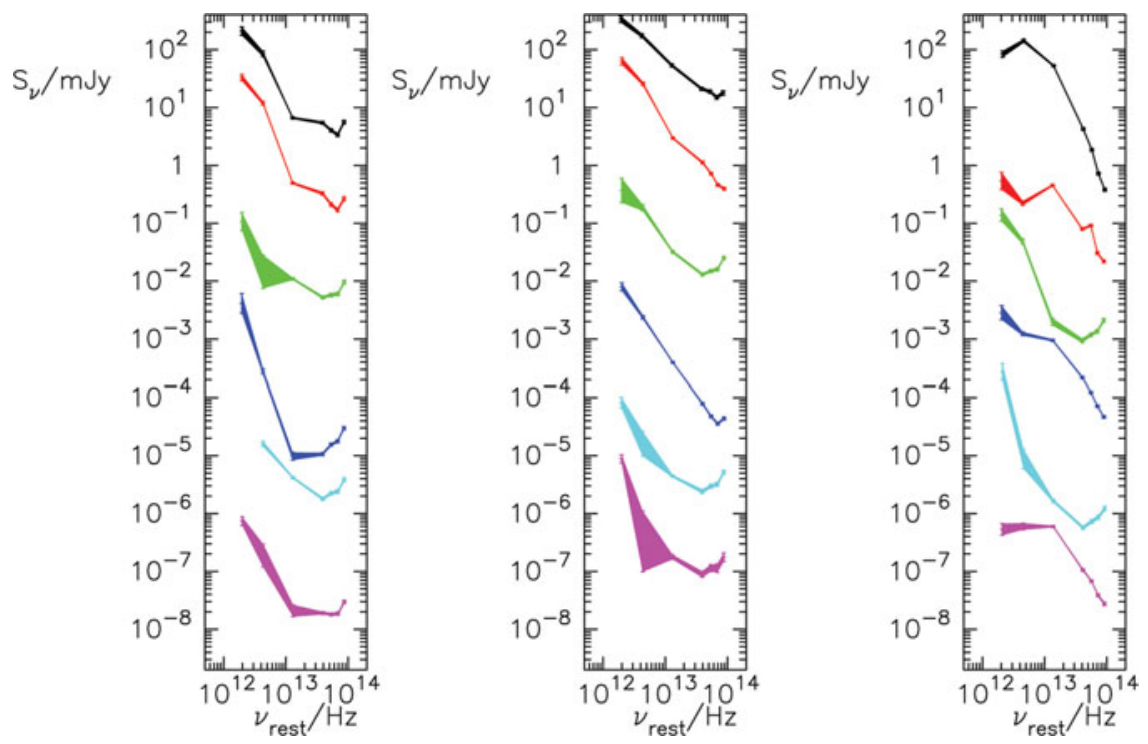

Figure 3. IR broad-band fluxes of the cores in representative low-power (left), medium-power (centre) and high-power (right) 3CRR radio galaxies. 178-MHz radio power increases downwards in each panel. The shape of the IR spectrum of a core is strongly related to the total radio power of the 3CRR source.

with IR colour more characteristic of a low-excitation source than a narrow-line-emitting source. This may indicate a misidentification of the core: 3C 326 has two compact radio sources near its centre, associated with two distinct galaxies, with different line-emitting properties. The optical characteristics of the northern galaxy are more characteristic of the radio structure (Rawlings et al. 1990), but the southern galaxy matches the behaviour of other 3CRR galaxies in its IR properties.

The distinction between high-power and low-power radio galaxies becomes clear when the full $3.6-160 \mu \mathrm{m}$ spectra of the 3CRR galaxies are considered (Fig. 3). In this representative set of spectra, the low-power objects (left panel) show far-IR emission from dust that peaks at lower frequencies than the high-power objects (right panel) in most cases. Residual stellar emission is also more evident at low and intermediate radio powers. The progressive change in shape of the spectra with increasing radio power strongly suggests the increasing presence of a hot dust component. The observation that there is a progression of properties also suggests that, for the majority of the 3CRR sources, the cores do not change dramatically on a shorter timescale than the lifetime of the large-scale radio structure. The lifetimes of the radio sources exceed $100 \mathrm{Myr}$, while the heat-loss times for dust near the cores are far shorter, $<1$ Myr.

The IR spectra shown in Fig. 3 can be fitted by the combination of a stellar continuum and dust with a range of temperatures and/or emissivities, with the characteristic temperature of the cool component being near $60 \mathrm{~K}$, and the hotter component being at about $300 \mathrm{~K}$. There is no evidence for a synchrotron component in these spectra the synchrotron components in 3CRR sources reported by Cleary et al. (2007) relate to quasars rather than radio galaxies.

It is notable that there are no strong correlations between the core radio and IR behaviours, though we find that the core radio flux density is a slightly better predictor of the core IR flux than is the total radio flux density. 


\section{Conclusions}

There are distinct differences between the core IR spectra, particularly longward of $24 \mu \mathrm{m}$, at different source powers. We believe that this indicates that the current behaviours of the cores in $3 \mathrm{CRR}$ radio galaxies are a reasonable representation of their average behaviours over the source lifetimes. However, in detail the relationship is not strong - perhaps indicating that the reservoir of material available for future activity is largely unrelated to the current nuclear activity of the sources, and that IR emission from the reservoir is a significant component of the measured fluxes.

The host galaxies show no strong IR peculiarities on scales resolvable with Spitzer in most cases, though some features associated with radio-source gas interactions have been noted. It is known from HST imaging that many of these objects show prominent dust lanes (e.g., de Koff et al. 1996; Martel et al. 1999; Sparks et al. 2000), but this dust probably produces long-wavelength IR emission that contributes to the $60-\mathrm{K}$ component that appears in the MIPS camera, and is not separated from the active cores.

Nevertheless, the angular resolution of Spitzer is sufficient to distinguish radio hot spots, jets, and jet/cloud interactions in a significant number of cases. Higher angular resolution, and improved sensitivity, would undoubtedly uncover more examples.

\section{Acknowledgements}

The full team working on the 3CRR low-redshift sample includes the authors of this short report and P. Green, H. Smith, B. Wilkes, S. Willner (CfA), C. Lawrence (JPL), P. Barthel (Groningen), E. Hooper (Wisconsin), D. Hines (STScI), and I. van Bemmel (ASTRON). We thank M. Ashby for his advice on processing the IRAC images. A fuller description of this work is being prepared for publication.

\section{References}

Cleary, K., Lawrence, C. R., Marshall, J. A., Hao, L., \& Meier, D. 2007, ApJ 660, 117

De Koff, S., Baum, S. A., Sparks, W. B., et al. 1996, ApJS 107, 621

Fazio, G. G., et al. 2004, ApJS 154, 10

Kraft, R. P., Birkinshaw, M., Hardcastle, M. J., et al. 2007, ApJ 659, 1008

Laing, R. A., Riley, J. M., \& Longair, M. S. 1983, MNRAS 204, 1519

Lanz, L., Bliss, A., Kraft, R. P., et al. 2011, ApJ 731, 52

Makovoz, D. \& Marleau, F. R. 2005, PASP 117, 1113

Martel, A. R., Baum, S. A., Sparks, W. B., et al. 1999, ApJS 122, 81

Rawes, J., Worrall, D. M., \& Birkinshaw, M. 2014, MNRAS in preparation

Rawlings, S., Saunders, R., Miller, P., \& Jones, M. E. 1990, MNRAS 246, 21P

Rieke, G. H., et al. 2004, ApJS 154, 25

Schuster, M. T., Marengo, M., \& Patten, B. M. 2005, Proc. SPIE 6270, 65

Sérsic, J. L. 1963, Bol. AAA 6, 41

Seymour, N., et al. 2007, ApJS 171, 353

Sparks, W. B., Baum, S. A., Biretta, J., Macchetto, F. D., \& Martel, A. R. 2000, ApJ 542, 667

Stern, D., et al. 2005, ApJ 631, 163

Tansley, D., Birkinshaw, M., Hardcastle, M. J., \& Worrall, D. M., 2000, MNRAS 317, 623 